\title{
Influencia de estrategias cooperativas en la solución de conflictos escolares
}

Influence of cooperative strategies in the solution of school conflicts

Influência de estratégias cooperativas na solução de conflitos escolares.

\section{ARTÍCULO GENERAL}

\section{Néstor Andrés Zamora García azamorag72@gmail.com https://orcid.org/0000-0001-7521-0326 IESPP “Don Bosco”, Lima - Perú}

\section{RESUMEN}

Actualmente en los centros educativos se evidencian situaciones que vulneran la integridad física y psicológica de los estudiantes. El objetivo del estudio es determinar la influencia de la aplicación de estrategias de aprendizaje cooperativo en la solución de conflictos en los estudiantes de secundaria de la Institución Educativa "Técnico Industrial" de Ayash Huaripampa, Ancash. Perú. Con un enfoque cuantitativo, de tipo explicativo, un diseño cuasiexperimental. La muestra no probabilística intencional representada por 120 estudiantes; se les aplicó un pre test, previamente al desarrollo del estímulo y finalmente un post test. Los resultados señalan que las puntuaciones promedio sobre la solución de conflictos en los estudiantes, se reflejó una superioridad el pos test (GC 10 - GE 17) respecto al promedio del pre test (GC $11-\mathrm{GE} 8)$. Concluyendo que la estrategia de aprendizaje cooperativo influye en la solución de conflictos de los estudiantes. Estos resultados demuestran que la aplicación de estrategias de aprendizaje cooperativo fomenta estilos de solución de conflictos que ayudan al cese de enfrentamientos violentos y la puesta en riesgo de acuerdos por puntos de vistas diversos.

Palabras clave: Aprendizaje, conflicto, cooperador.

\section{ABSTRACT}

Currently in educational centers there are situations that violate the physical and psychological integrity of students. The objective of the study is to determine the influence of the application of cooperative learning strategies in conflict resolution in high school students of the "Industrial Technician" Educational Institution of Ayash Huaripampa, Ancash. Peru. With a quantitative, explanatory approach, a quasi-experimental design. The intentional non-probabilistic sample represented by 120 students; a pre-test was applied to them, prior to the development of the stimulus and finally a post-test. The results indicate that the average scores on the solution of conflicts in the students, reflected a superiority in the post test (GC 10 - GE 17) with respect to the average of the pre-test (GC 11 - GE 8). Concluding that the cooperative learning strategy influences the conflict resolution of students. These results show that the application of cooperative learning strategies fosters conflict resolution styles that help to stop violent confrontations and put agreements at risk due to different points of view.

Keywords: Learning, conflict, cooperative.

\section{RESUMO}

Atualmente nos centros educacionais existem situações que violam a integridade física e psicológica dos alunos. O objetivo do estudo é determinar a influência da aplicação de estratégias de aprendizagem cooperativa na resolução de conflitos em alunos do ensino médio da Instituição Educacional "Técnico Industrial" de Ayash Huaripampa, Ancash. Peru. Com uma abordagem quantitativa, explicativa, um design quase-experimental. A amostra intencional não probabilística representada por 120 alunos; a eles foi aplicado um pré-teste, antes do desenvolvimento do estímulo e por último um pós-teste. Os resultados indicam que as notas médias sobre a solução de conflitos dos alunos refletiram uma superioridade no pósteste (GC 10 - GE 17) em relação à média do pré-teste (GC 11 - GE 8). Concluindo que a estratégia de aprendizagem cooperativa influencia na resolução de conflitos dos alunos. Esses resultados mostram que a aplicação de estratégias de aprendizagem cooperativa promove estilos de resolução de conflitos que ajudam a frear confrontos violentos e colocam em risco os acordos devido a diferentes pontos de vista.

Palavras-chave: Aprendizagem, conflito, cooperativo. 


\section{Introducción}

Las diferentes concepciones culturales alrededor de los conflictos escolares, están estructuradas desde paradigmas vinculados con violencia, como elementos negativos que buscan excluir en cualquier contexto donde se presente. Las instituciones educativas de nuestro país, Latinoamérica, y porque no decir en el contexto global; no son ajenas a esta situación, pues en ella se presentan desacuerdos, enfrentamientos, tensiones y hostilidades, propias de nuestra naturaleza humana.

De acuerdo a expuesto anteriormente, se puede sostener que todas las relaciones sociales tienen un elemento de conflicto; lo que hace especular que estos son inherentes al ser humano. Por tanto, su eliminación o erradicación dentro de la vida de los seres humanos conllevaría a que las dinámicas de interacción social fueran estériles. Desde estas consideraciones debemos de enfocarnos en una resignificación del conflicto escolar desde una perspectiva proactiva para la construcción de la convivencia escolar buscando darle un nuevo significado al conflicto desde su interpretación y comprensión.

Para ello esta investigación gira en torno a tres ejes fundamentales como son: la resignificación, el conflicto, y la convivencia. Los cuales son analizados desde las perspectivas teóricas de: Lewis Coser (1961), Marc Howard (1995), Julien Freund (1995) y Ralf Dhrendorf (1996), Vinyamata, Eduard (2001), Fisas, V. (2001) y Girar, K. (1997); quienes realizan aportes al conflicto. Los planteamientos de estos autores aportan elementos fundamentales para entender los problemas y conflictos convivenciales.

Los aspectos anteriores son el resultado de un riguroso estudio, de las problemáticas que afectan el contexto escolar de la Institución Educativa “Técnico Industrial” de Ayash Huaripampa y que fueron analizadas a través de técnicas cuantitativas como la escala de valoración diseñada por el Minedu (2019), que permitieron analizar las diferentes situaciones de conflicto que se viven en el entorno escolar.

Finalmente, "numerosos estudios correlacionan la competencia para resolver conflictos con la capacidad de autorregulación emocional" (Galvis, 2014). Debido a la interconexión de nuestro mundo emocional, no se puede desvincular el aprendizaje de la resolución de conflictos de otras destrezas sociales y emocionales (Soriano, 2006); Sin embargo, tiene sentido analizar separadamente cada una de ellas para hacer una 
evaluación competencial, entendida como la selección y combinación pertinente de conocimientos, habilidades, destrezas, actitudes, valores y normas que permiten dar respuesta a una situación concreta y en un contexto determinado (Perrenoud, 2004), que permita una gestión adecuada de la conflictividad en los centros educativos.

\section{Solución de conflictos escolares.}

Existe una concepción muy amplia, que es la de ver el conflicto como algo negativo y, por tanto, algo a rehuir. Por eso, al abordar el tema del conflicto, es imprescindible hacer un recorrido por los diferentes autores y especialistas. (Vinyamata, 2001, pág. 129), sostiene que: El conflicto es definido como lucha, desacuerdo, incompatibilidad aparente, confrontación de intereses, percepciones o actitudes hostiles entre dos o más partes. El conflicto es connatural con la vida misma, está en relación directa con el esfuerzo por vivir. Los conflictos se relacionan con la satisfacción de las necesidades, se encuentra en relación con procesos de estrés y sensaciones de temor y con el desarrollo de la acción que puede llevar o no hacía comportamientos agresivos y violentos.

El autor señala que el conflicto es connatural a la vida, esto implica que siempre habrá conflictos por que todos buscamos satisfacer las necesidades básicas, y esto genera conflictos. Sin embargo, como señala (Fisas, 2001, pág. 30), que puede existir conflicto sin violencia.

Es un proceso interactivo que se da en un contexto determinado. Es una construcción social, una creación humana, diferenciada de la violencia (puede haber conflictos sin violencia, aunque no violencia sin conflicto), que puede ser positivo o negativo según cómo se aborde y termine, con posibilidades de ser conducido, transformado y superado.

Por su parte, Entelman(2002, pág. 173), sostiene que las actitudes y conductas se pueden modificar, si se toman decisiones estratégicas.

El conflicto es un proceso dinámico, sujeto a la permanente alteración de todos sus elementos. A medida que se desarrolla su devenir cambian las percepciones y las actitudes de los actores que, en consecuencia, modifican sus conductas, toman nuevas decisiones estratégicas sobre el uso de los recursos que integran su poder y, a menudo, llegan a ampliar, reducir, separar o fusionar sus objetivos. 
Tal como hemos cotejado, las definiciones guardan ciertas similitudes. Por lo tanto, un aspecto común: el conflicto es una contraposición a alguien o algo. Los autores citados, hablan de los conflictos para indicar un hecho de lucha o desacuerdo. Así comprendemos que el término "conflicto" se refiere a las fuerzas o posiciones contrarias que en un determinado momento se encuentran y se colisionan, pudiendo ser por interés común o por discrepancias.

El conflicto sirve de respaldo para el cambio; a partir de una situación explícita conflictiva se desencadena una serie de reacciones positivas o negativas. Lo que está claro es que cuando uno entra en conflicto con alguien de su ambiente es porque en cierta parte se siente amenazado desde sus valores sociales, morales, culturales, religiosos, aspectos que conciernen al individuo o al grupo. Por consiguiente, un conflicto interpersonal existe cuando se da cualquier tipo de posicionamiento o actividad incompatible entre dos o más sujetos, lo cual no es necesariamente negativo.

Pensar en definiciones y asociaciones puede dar una primera base más firme para analizar el conflicto, pero es sólo un pequeño paso inicial hacia una comprensión positiva y productiva del mismo. Para ver el conflicto claramente, debemos ser capaces de ver más allá de nuestras respuestas más familiares y habituales.

Los sentimientos, pensamientos, reacciones físicas y conductas en torno al conflicto surgen, de las creencias, supuestos y experiencias con los que fuimos criados. Saber que el conflicto es normal y potencialmente beneficioso, no es suficiente para cambiar la creencia de toda una vida acerca de que el conflicto es peligroso, ni para alterar una respuesta incorporada y establecida de evitar el conflicto. ¿Cómo aprendemos a ver el conflicto en profundidad y con perspectiva, y de maneras que lleven a experiencias positivas? ¿Cómo aprendemos a ver los conflictos claramente a pesar del temor y la ansiedad que a menudo oscurecen las situaciones de conflicto? La disciplina de la solución de conflictos ofrece una variedad de lentes a través de las cuales mirar el conflicto. Estos lentes se convierten en herramientas para aprender a abandonar antiguas creencias, ideas y hábitos, y ver con ojos renovados.

Pueden ayudar a tener una visión más amplia, logrando enfocar más nítidamente el conflicto y aportando una perspectiva con mayor distancia. La conciencia de que existen distintas maneras de ver un conflicto puede evitar que nos quedemos trabados en una sola 
visión improductiva. Hay maneras de ver un conflicto que exploraremos aquí. Las llamamos orígenes, fuentes, tipos, creencias y postura, cada una tiene una o dos cuestiones centrales para guiar nuestra mirada.

Estrategia cooperativa como actividad para trabajar en la solución de conflictos

$\mathrm{Al}$ inicio del bimestre del año lectivo se propuso el aprendizaje cooperativo, como una estrategia didáctica que permite adquirir distintos tipos de competencias; como la escucha asertiva de las diversas opiniones que se expresan, la solución de conflictos o la expresión de diferentes opiniones de manera cortés. En tal sentido, los estudiantes tomarán conciencia, que se pretende descubrir y potenciar las habilidades personales y grupales en la solución de conflictos existentes dentro de la Institución Educativa. Para lo cual, seleccionamos situaciones cotidianas que llevan a tener una mirada precisa, cercana, subjetiva y, a la vez, objetiva de la situación (Sánchez, 2018). Para que, semanalmente cada equipo o grupo de trabajo tuviera que preparar un debate cooperativo sobre el tema o situación seleccionado. Teniendo la oportunidad de liderar, controlar el tiempo, gestionar las distintas intervenciones e impulsar la participación de cada uno de los miembros del equipo de trabajo.

El uso de la estrategia del aprendizaje cooperativo, nos permitió dividir la muestra en pequeños grupos de 05 ó 06 estudiantes, para desarrollar, pensar y discutir: la identificación de conflictos, búsqueda de alternativas de solución, Analizar y evaluar las alternativas de solución y la Puesta en práctica de las alternativas de solución. Cada uno de los miembros del equipo juegan un rol importantísimo, establecido por Johnson, D. W., Johnson, R. T. \& Holubec, E. (1996):

- Compendiador: se encarga de resumir las principales conclusiones o respuestas generadas por el grupo.

- Inspector: se asegurará que todos los miembros puedan decir explícitamente como llegaron a las conclusiones o respuestas.

- Entrenador: corrige los errores de las explicaciones o resúmenes de los otros miembros. 
- Narrador: pide a los integrantes del grupo que relacionen los nuevos conceptos y estrategias con el material aprendido previamente.

- Investigador-Mensajero: consigue los materiales que el grupo necesita. Se comunica con los otros grupos y con el profesor.

- Registrador: escribe las decisiones del grupo y edita el reporte del trabajo.

- Animador: refuerza las contribuciones de los miembros.

- Observador: cuida que el grupo esté colaborando de manera adecuada. Dependiendo del tamaño del grupo un alumno puede asumir uno a más funciones.

Lo mencionado anteriormente, facilita y promueve el aprendizaje autónomo, lo que enmarca la curricular actual. Esta metodología conduce al estudiante a ser crítico sobre la realidad en la que vive y a transformarla.

\section{RESULTADOS}

Resultados según test

Tabla 4

Puntajes obtenidos en el pre test solución de conflictos según grupo control y experimental

\begin{tabular}{|c|c|c|c|c|c|}
\hline \multirow{3}{*}{$\begin{array}{l}\text { Nivel de } \\
\text { Solución } \\
\text { de } \\
\text { conflictos }\end{array}$} & \multirow{3}{*}{ Intervalo } & \multicolumn{4}{|c|}{ Grupo } \\
\hline & & \multicolumn{2}{|c|}{ Control } & \multicolumn{2}{|c|}{ Experimental } \\
\hline & & fi & $\%$ & fi & $\%$ \\
\hline Inicio & 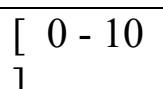 & 27 & $46 \%$ & 45 & $74 \%$ \\
\hline Proceso & [ $11-13$ & 24 & $41 \%$ & 8 & $13 \%$ \\
\hline $\begin{array}{l}\text { Logro } \\
\text { previsto }\end{array}$ & $\begin{array}{l}{[14-} \\
17]\end{array}$ & 8 & $14 \%$ & 8 & $13 \%$ \\
\hline $\begin{array}{l}\text { Logro } \\
\text { destacado }\end{array}$ & {$[18-20$} & 0 & $0 \%$ & 0 & $0 \%$ \\
\hline Total & & 59 & $100 \%$ & 61 & $100 \%$ \\
\hline Mediana & & 11 & & 8 & \\
\hline
\end{tabular}

Fuente: Resultados ficha de observación en estudiantes de educación secundaria de la Institución Educativa "Técnico Industrial” de Ayash Huaripampa - San Marcos, 2020. 


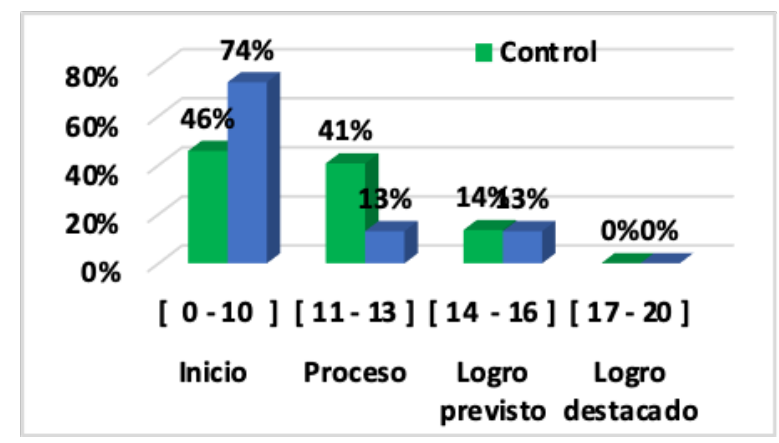

Gráfico 1. Gráfico de barra de los niveles de solución de conflictos del grupo control y experimental según pre test

Según la tabla 4 y el gráfico 1 presenta el nivel de solución de conflictos antes de la aplicación de del aprendizaje cooperativo en el logro del de solución de problemas el diagnostico presenta los resultados para el grupo experimental pues el $74 \%$ de los estudiantes se encuentran en un nivel de inicio en solución de conflictos, el 13\% en un nivel de proceso y logro previsto, ninguno de los estudiantes logro previsto, demostrando un el bajo nivel para solucionar problemas en estudiantes de educación secundaria de la Institución Educativa “Técnico Industrial” de Ayash Huaripampa - San Marcos, 2020.

De Igual manera los resultados muestran resultados parecidos para el grupo control un $46 \%$ de los estudiantes se encuentran en un nivel de inicio, en solución de conflictos, seguido del $41 \%$ alcanzan el nivel proceso finalmente solo el $14 \%$ alcanzo el nivel logro previsto para la solución de conflictos en estudiantes de educación secundaria de la Institución Educativa "Técnico Industrial” de Ayash Huaripampa - San Marcos, 2020. Los grupos muestran niveles parecidos para el nivel de logro para solución de conflictos

\section{Tabla 5}

Puntajes obtenidos en el postest solución de conflictos según grupo control y experimental

\begin{tabular}{llllll}
\hline Nivel de & & \multicolumn{2}{l}{ Grupo } & \\
\cline { 3 - 6 } $\begin{array}{l}\text { Solución } \\
\text { de }\end{array}$ & Intervalo & Control & \multicolumn{2}{l}{ Experimental } \\
\cline { 3 - 6 } conflictos & fi & $\%$ & fi & $\%$ \\
\hline Inicio & {$[0-10$} & 51 & $86 \%$ & 0 & $0 \%$
\end{tabular}




\begin{tabular}{llllll} 
Proceso & {$[11-13$} & 6 & $10 \%$ & 0 & $0 \%$ \\
$\begin{array}{l}\text { Logro } \\
\text { previsto }\end{array}$ & {$[14-$} & 2 & $3 \%$ & 50 & $82 \%$ \\
$\begin{array}{l}\text { Logro } \\
\text { destacado }\end{array}$ & {$[18-20$} & 0 & $0 \%$ & 11 & $18 \%$ \\
\hline Total & 59 & $100 \%$ & 61 & $100 \%$ \\
\hline Mediana & 10 & & 17 & \\
\hline
\end{tabular}

Fuente : Resulatdos ficha de observacion en estudiantes de educación secundaria de la Institución Educativa "Técnico Industrial” de Ayash Huaripampa - San Marcos, 2020.

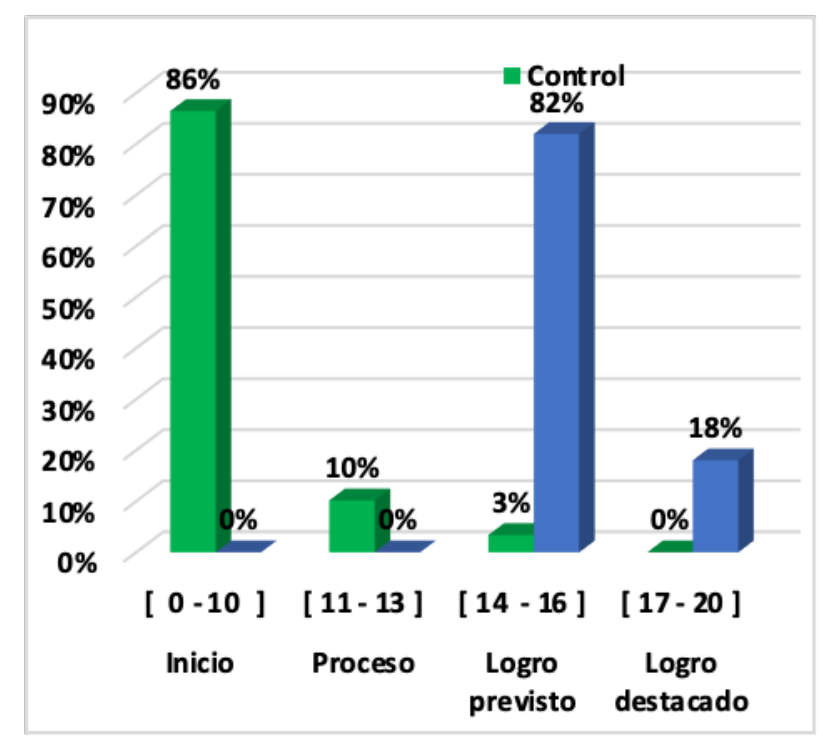

Gráfico 2. Gráfico de barra de los niveles de solución de conflictos del grupo control y experimental según pos test

Según la tabla 5 y el gráfico 2 presenta el nivel de solución de conflictos después de la aplicación de del aprendizaje cooperativo en el logro del de solución de problemas para el grupo experimental los resultados muestran resultados favorables en el nivel de logro para solución de conflictos tenemos el $82 \%$ de los estudiantes alcanzaron el nivel logro previsto en solución de conflictos, seguido del 18\% alcanzaron el nivel logro destacado, se superaron los niveles inicio y proceso para la solución de conflictos demostrando la influencia del aprendizaje cooperativo para la solución de conflictos en los estudiantes de educación secundaria de la Institución Educativa "Técnico Industrial" de Ayash Huaripampa - San Marcos, 2020. 
De Igual manera los resultados muestran resultados parecidos para el grupo control respecto al pretest así tenemos el 86\% de los estudiantes mantienen el nivel de inicio, en solución de conflictos, seguido del 10\% alcanzan el nivel proceso finalmente solo el 3\% alcanzo el nivel logro previsto para la solución de conflictos en estudiantes de educación secundaria de la Institución Educativa "Técnico Industrial" de Ayash Huaripampa - San Marcos, 2020. Los grupos muestran diferencias muy significativas para el nivel de logro para solución de conflictos demostrando nuestra hipótesis planteada el aprendizaje cooperativo influencia en la solución de conflictos de los estudiantes de educación secundaria de la Institución Educativa "Técnico Industrial” de Ayash Huaripampa - San Marcos, 2020.

\section{Prueba de hipótesis}

Para contrastar la hipótesis de la variable resolución de conflictos se utilizó la prueba Z de U de Mann-Whitney (Prueba no paramétrica) para muestras independientes (grupo control y grupo experimental) con cuyos datos han sido medidos en una escala de Likert. Presentando alta fiabilidad según la prueba de fiabilidad alfa de Crombach pues los resultados tienen distribución libre según la prueba de normalidad de Shapiro Wilk, Para contrastar la prueba de hipótesis explicar la influencia del aprendizaje cooperativo en la solución de conflictos de los estudiantes de educación secundaria de la Institución Educativa "Técnico Industrial” de Ayash Huaripampa - San Marcos, 2020.

La aproximación a la normal, $\mathrm{z}$, cuando tenemos muestras lo suficientemente grandes viene dada por la expresión:

$$
Z=\frac{U-\mu_{U}}{\sigma_{U}}
$$

Donde $\mu \mathrm{U}$ y $\sigma \mathrm{U}$ son la media y la desviación estándar de U si la hipótesis nula es cierta, y vienen dadas por las siguientes fórmulas:

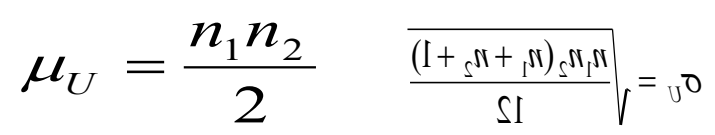


Se rechaza $\mathrm{H} 0$ si

$\mathrm{p}($ valor $)<\alpha$

$\mathrm{Zc}<\mathrm{Zt}$

Conclusión: Se acepta la H1; Hipótesis de nuestra investigación

Tabla 10

Prueba de hipótesis para eexplicar la influencia del aprendizaje cooperativo en la solución de conflictos de los estudiantes de educación secundaria de la Institución Educativa "Técnico Industrial” de Ayash Huaripampa - San Marcos, 2020.

\begin{tabular}{ll}
\hline Estadísticos de prueba & \\
\hline Prueba & $\begin{array}{l}\text { Solución de } \\
\text { conflictos }\end{array}$ \\
\hline U de Mann-Whitney & 29.000 \\
W de Wilcoxon & 1799.000 \\
Z & -9.432 \\
Sig. asintótica (bilateral) & .000 \\
\hline
\end{tabular}

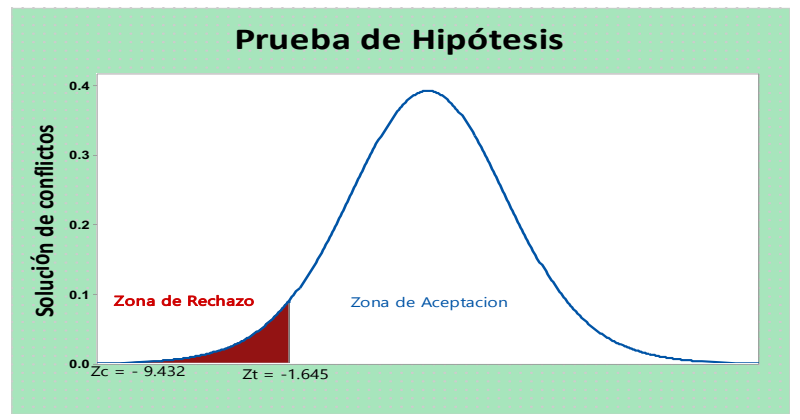

Figura 11: Representación gráfica de U de Mann-Whitney

\section{Hipótesis estadística}

Ho = El aprendizaje cooperativo no influye en la solución de conflictos de los estudiantes de educación secundaria de la Institución Educativa "Técnico Industrial" de Ayash Huaripampa - San Marcos, 2020. 
H1= El aprendizaje cooperativo influye en la solución de conflictos de los estudiantes de educación secundaria de la Institución Educativa "Técnico Industrial” de Ayash Huaripampa - San Marcos, 2020.

\section{Decisión}

Se rechaza $\mathrm{HO} \mathrm{p}$ (valor) $<0.05$

$$
\mathbf{Z c}<\mathbf{Z t}
$$

A continuación, se presentan los resultados del postest en la comparación de las medianas de las puntuaciones de solución de conflictos de los estudiantes de educación secundaria de la Institución Educativa “Técnico Industrial” de Ayash Huaripampa - San Marcos, 2020. Después de la aplicación de aprendizaje cooperativo, en el grupo experimental los resultados muestran la superioridad de la mediana del grupo experimental (17) respecto a la mediana del grupo control (10), diferencia justificada mediante la prueba $\mathrm{U}$ de Mann-Whitney $(\mathrm{Z})$ para muestras independientes con $(\mathrm{P}$ valor $<$ 0.05), el valor Zc (calculada) = 9,432 menor que el valor teórico Zt (tabular)= -1,645, para un nivel de significancia de $(\alpha=0,05)$, ello implica rechazar la hipótesis nula (H0). Y aceptar la hipótesis alterna (H1). Concluimos que el aprendizaje cooperativo influye en la solución de conflictos de los estudiantes de educación secundaria de la Institución Educativa “Técnico Industrial” de Ayash Huaripampa - San Marcos, 2020.

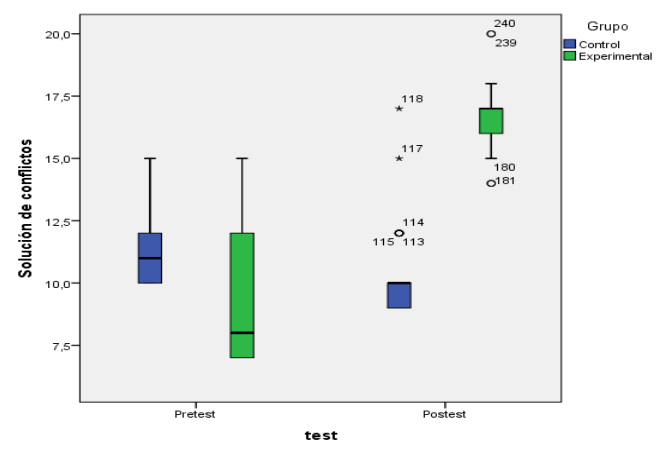

\section{DISCUSIÓN}

La influencia del aprendizaje cooperativo en el nivel de logro solución de conflictos de los estudiantes de educación secundaria de la Institución Educativa "Técnico Industrial" de Ayash Huaripampa - San Marcos, 2020, Permitió identificar el problema, 
la búsqueda de alternativas, analizar y evaluar alternativas de solución, puesta en práctica, luego de la aplicación del aprendizaje cooperativa en el logro de la solución de conflictos.

Al respecto, Johnson \& Johnson (2004), menciona Trabajar de forma cooperativa favorece que el estudiante tome conciencia de sus propias emociones al tener que tomar decisiones, negociar, gestionar conflictos en los grupos, desarrollar la capacidad crítica, entre otras. Nuestra experiencia llevando a cabo actividades cooperativas en la enseñanza universitaria nos ha hecho tomar conciencia de que la cooperación lleva consigo conflictos que han de resolverse en los grupos de trabajo (Martínez, 2016). En este sentido, el conflicto en un entorno cooperativo ofrece la posibilidad de crecer de manera personal y grupal si se le da el enfoque adecuado pues el estudiante tiene que reflexionar y pensar para encontrar soluciones.

Ahora bien, los resultados de la hipótesis general, se reflejó superioridad de la mediana del grupo experimental (17) respecto a la mediana del grupo control (10), diferencia justificada para un nivel de significancia de $(\alpha=0,05)$, ello implica rechazar la hipótesis nula (H0). Concluimos que el aprendizaje cooperativo influye en la solución de conflictos de los estudiantes de educación secundaria de la Institución Educativa "Técnico Industrial" de Ayash Huaripampa - San Marcos, 2020. Esto concuerda con Chávez (2012).

Asimismo, el resultado de la hipótesis especifica 01 respecto a la dimensión Identifica el conflictose reflejó superioridad de la mediana del grupo experimental (18) respecto a la mediana del grupo control (7), diferencia justificada para un nivel de significancia de $(\alpha=0,05)$, ello implica rechazar la hipótesis nula (H0). Concluimos que el aprendizaje cooperativo influye el aprendizaje cooperativo influye en Identifica el conflicto de los estudiantes de educación secundaria de la Institución Educativa "Técnico Industrial" de Ayash Huaripampa - San Marcos, 2020. esto se ve reflejado en el gráfico de caja y bigotes.

Ahora bien, los resultados de la hipótesis especifican 02 respecto a la dimensión Búsqueda de alternativa de solución se reflejó superioridad en su mediana del grupo experimental (17) respecto a la mediana del grupo control (8), diferencia justificada para un nivel de significancia de $(\alpha=0,05)$, ello implica rechazar la hipótesis nula (H0). Concluimos que el aprendizaje cooperativo influye en la Búsqueda de alternativa de 
solución de los estudiantes de educación secundaria de la Institución Educativa "Técnico Industrial" de Ayash Huaripampa - San Marcos, 2020. esto se ve reflejado en el gráfico de caja y bigotes.

Ahora bien, los resultados de la hipótesis especifican $\mathbf{0 3}$ respecto a la dimensión Analiza y evalúa las alternativas de solución se reflejó superioridad en su mediana del grupo experimental (17) respecto a la mediana del grupo control (12), diferencia justificada para un nivel de significancia de $(\alpha=0,05)$, ello implica rechazar la hipótesis nula (H0). Concluimos que el aprendizaje cooperativo influye en la Analiza y evalúa las alternativas de solución de los estudiantes de educación secundaria de la Institución Educativa "Técnico Industrial" de Ayash Huaripampa - San Marcos, 2020. esto se ve reflejado en el gráfico de caja y bigotes.

Ahora bien, los resultados de la hipótesis especifican 04 respecto a la dimensión Puesta en práctica de las alternativas de solución se reflejó superioridad en su mediana del grupo experimental (16) respecto a la mediana del grupo control (11), diferencia justificada para un nivel de significancia de $(\alpha=0,05)$, ello implica rechazar la hipótesis nula (H0). Concluimos que el aprendizaje cooperativo influye en Puesta en práctica de las alternativas de solución de los estudiantes de educación secundaria de la Institución Educativa "Técnico Industrial" de Ayash Huaripampa - San Marcos, 2020. esto se ve reflejado en el gráfico de caja y bigotes.

\section{Conclusiones}

\section{General:}

El aprendizaje cooperativo influye en la solución de conflictos de los estudiantes de educación secundaria de la Institución Educativa "Técnico Industrial" de Ayash Huaripampa - San Marcos, 2020.

\section{Específicos:}

la interdependencia positiva del aprendizaje cooperativo influye en la identificación de conflictos de los estudiantes de educación secundaria de la Institución Educativa “Técnico Industrial” de Ayash Huaripampa - San Marcos, 2020. 
la responsabilidad individual y grupal del aprendizaje influye cooperativo en la búsqueda de alternativas de solución de los conflictos. de la Institución Educativa “Técnico Industrial” de Ayash Huaripampa - San Marcos, 2020.

las habilidades del aprendizaje cooperativo influyen en el análisis y evaluación de las alternativas de solución de conflictos de los conflictos. de la Institución Educativa “Técnico Industrial” de Ayash Huaripampa - San Marcos, 2020.

El procesamiento grupal del aprendizaje cooperativo influye en la puesta en práctica de las alternativas de solución de conflictos de los conflictos. de la Institución Educativa “Técnico Industrial” de Ayash Huaripampa - San Marcos, 2020.

\section{REFERENCIAS}

Coser, 1. (1961). Las Funciones del Conflicto. Mexico: Fondo de Cultura Económica.

Dahrendorf, R. (1996). Elementos para una teoría del conflicto social: en sociedad y libertad. Madrid: Tecnos.

Entelman, R. F. (2002). Teoría de los Conflictos. Barcelona: Gedisa.

Fisas, V. (2001). Cultura de paz y gestión de Conflictos. Barcelona: Icaria.

Freund, J. (1995). Sociología del conflicto. Madrid: Editorial del Ministerio de Defensa.

Galvis, R. I. (2014). Las neuronas espejo y el desarrollo de la empatía frente a la agresión y el conflicto en la escuela. Praxis pedagógica, 15,43-53.

Girard, K. \&. (1997). Resolución de conflicctos en las escuelas-. España: Granica S.A.

Howard, M. (1995). La cultura del conflicto: las diferencias interculturales en la práctica de la violencia. Barcelona: Paidós Ibérica.

Johnson D.W. \& johnson, R. (2004). Assessing in grous. Promoting group responssibility and individual accountability. California: Corwin Press.

Johnson, D. W. (1996). Apprendimento cooperativo in classe. Migliorare il clima emotivo e il rendimento. Trento. 
Martínez, M. (2016). Hacia una resolución efectiva de conflictos en las aulas universitarias: Ejemplos a través del debate cooperativo. Zona Próxima, 103-114.

Minedu. (12 de Febrero de 2019). www.minedu.gob.pe. Obtenido de http//www.minedu.gob.pe/siagie

Perrenoud, P. (2004). Nuevas competencias para enseñar. Barcelona: Graó.

Sánchez, M. L. (2018). El conflicto en el aula de educación básica: Experiencias y modelos de solución. México: Universidad Pedagógica Nacional.

Soriano, E. (2006). Competencias ciudadanas en el alumnado de segundo ciclo de educación secundaria obligatoria de Almería. Revista de Investigación Educativa, 24, 199-146.

Vinyamata, E. (2001). Conflictología. Barcelona: Ariel 